\title{
Blowing up warped disks
}

\author{
V. Icke $e^{1, \star}$ \\ Sterrewacht Leiden, Postbus 9513, 2300 RA Leiden, The Netherlands
}

Received 12 December 2002 / Accepted 15 May 2003

\begin{abstract}
Stars do not go gently: even low-mass stars such as our Sun blow up in the end, seeding space with the elements of which we are made. Usually, the resulting nebulae show a pronounced bipolar or even multipolar shape. Balick's "generalized interacting-winds" model posits that this is due to an interaction between a very fast tenuous outflow, and a disk-shaped denser atmosphere left over from an earlier slow phase of mass loss. Analytical and numerical work shows that this mechanism can explain cylindrically symmetric nebulae. However, many circumstellar nebulae have a "multipolar" or "point-symmetric" shape. I demonstrate that these seemingly enigmatic forms can be easily reproduced by a two-wind model in which the confining disk is warped, as is expected to occur in irradiated disks. Large-scale explosions in other non-planar disks, such as might occur in active galaxies, are expected to show similar patterns.
\end{abstract}

Key words. stars: planetary nebulae - hydrodynamics

\section{Introduction}

In the final phases of stellar evolution, low mass stars, such as our Sun, first swell up and shed a dense, cool wind in the "asymptotic giant branch" (AGB) phase. This episode is followed by a fast, tenuous wind that is driven by the exposed stellar core, which will later become a white dwarf. Surprisingly, the nebulae resulting from this expulsion phase are rarely spherical. More often they show a pronounced bipolar shape. Balick (1987) proposed that such forms arise due to an interaction between a slow disk-shaped inner AGB nebula and the fast "last gasp" of the star. Analytical (Icke 1988; Icke et al. 1989) and numerical (Soker \& Livio 1989; Icke 1991; Mellema et al. 1991) work shows that this mechanism works very well (for an up-to-date review, see Balick \& Frank 2002).

The shape of the dense gas around the star is assumed to be a disk or a toroid. Its origin remains unexplained; Soker \& Livio (1994) have argued that the disk is formed when compact binaries coalesce, even though the statistics of binary stars make this questionable. The scenario might still work, though, because it also applies to the re-absorption of planetary systems (including the "super-Jupiters" found around nearby stars) when the central star dies. This is, as it were, star formation run in reverse. Alternatively, the recently observed strong magnetic fields may play a role (Vlemmings 2002, 2002).

\section{Point-symmetric nebulae}

My work on cylindrically symmetric nebulae showed (Icke 1988, 1991, 1992) that sharply collimated bipolar jets are a frequent and natural by-product of the two-wind interaction.

\footnotetext{
* e-mail: icke@strw.LeidenUniv.nl
}

However, many circumstellar nebulae have a "multipolar" or "point-symmetric" shape (Schwarz 1993; Sahai \& Trauger 1998). Upon a challenge leveled at the theoretical community by Sahai (2003), it occurred to me that the nebulae that are formed in the disk-wind interaction would naturally acquire the observed symmetric multipolar shape if the disk that confines the fast wind is warped, instead of symmetric under reflection about the equatorial plane. In binary stars such warps might be common, due to various torques in the system, but few pointsymmetric nebulae have binary cores. Many mechanisms have been proposed for warping a disk surrounding a single star. The most interesting one for my purposes invokes radiative instability (Petterson 1977; Iping \& Petterson 1990; Pringle 1996; Maloney et al. 1996). I investigated the collision between a fast wind and a disk of the Maloney-Begelman-Pringle (MBP 1996) type:

$z=\operatorname{ar} \sin \left(\frac{1}{\sqrt{r}} \sin \sqrt{r}\right) \sin (\phi+b \sqrt{r})$.

As in the cylindrical case, the orbital motion of the disk and the precession do not matter much if the central wind is very supersonic.

A study of the literature revealed that Livio \& Pringle had already proposed $(1996,1997)$ that the precession of warped disks might be responsible for point-symmetric nebulae. They proved conclusively that the various physical scales for mass, accretion, luminosity and precession match the observations; the production of the nebulae proper they attributed to an unspecified "jet" mechanism. Observations of many bipolar nebulae with "ansae" (e.g. NGC 3242, NGC 7009) and "FLIERS" (e.g. NGC 6751, NGC 7662) leave little doubt that jets are occasionally formed during the evolution of some aspherical PNs, 


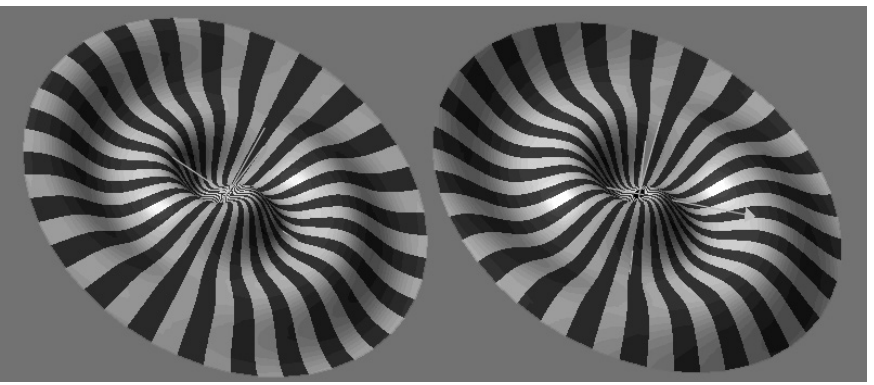

Fig. 1. Warped disk surfaces, showing on the left the MaloneyBegelman-Pringle cross section. On the right the sinusoidal shape.

probably in the late post-AGB phase, before the fast wind has switched on. But the nebulae presented by Sahai \& Trauger (1998) do not seem to resemble such shapes very much.

While leaving open the possibility that jets may be responsible for additonal structures, as in the case of the "ansae", I show that the interaction between a warped disk and a spherically symmetric wind suffices. The lobes of point-symmetric nebulae (Schwarz 1993; Sahai \& Trauger 1998) look as if they were produced almost simultaneously. This is hard to do in the case of a precessing jet, which would make a corkscrew-like nebula of a type not readily apparent in post-AGB shells, although some features agree with precession (Schwarz 1993). Another possibility is invoking magnetic fields (García-Segura et al. 1999), which are known to exist in the circumstellar masers (Vlemmings 2002) but these are not needed for the mechanism proposed here.

\section{Two-wind flow in a warped disk}

To do the job properly, one should use a three-dimensional hydrocode, but in order to provide a quick proof-ofprinciple I adapted my two-dimensional code to the problem at hand. The computations presented here were performed using my FCT/LCD hydrocode (Boris \& Book 1973, 1976; Icke 1991), programmed in C, running the MetroWerks CodeWarrior compiler on an Apple PowerBook G3 and a PowerPC/G4. Graphic processing was carried out with the NIH Image freeware package distributed by the U.S. National Institute of Health. I constructed a "warped wedge" with a cross section obeying the MBP solution, and set off a spherical wind at its origin. The flow equations were cast in dimensionless form, to allow scaling afterwards in applications to a variety of astrophysical conditions. Thus, I have opted not to model the energy loss of the gas by means of a cooling function, but to use instead a gas equation of state that is made highly compressible ("momentum-driven") by using a small Poisson index $(\gamma=1.1)$. The main disk parameters are the tilt of the innermost piece of the disk, the opening angle of the wedgeshaped cross section of the disk, the density contrast between disk and wind, and the speed of the fast wind (wind parameters refer to the state at the point where the wind leaves the central sphere, prescribed as an inner boundary condition). I explored a large range of disk parameters and found that multipolar pointsymmetric shells are formed in all cases.

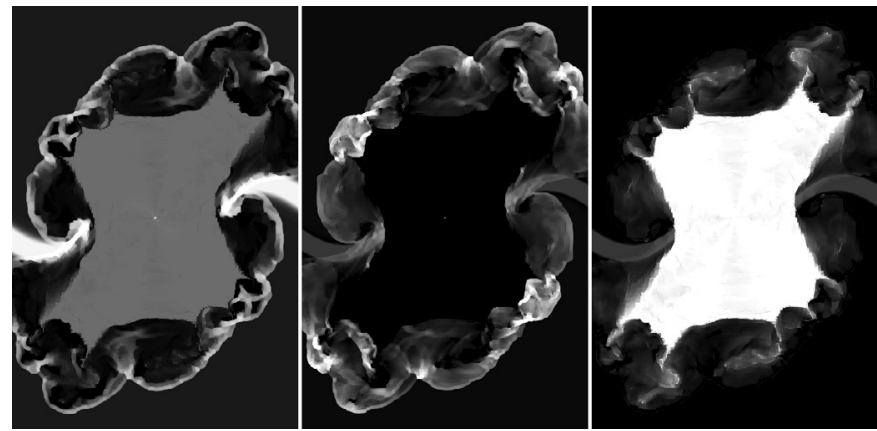

Fig. 2. Snapshot from a simulated interaction between a fast wind and a "warped wedge" with MBP cross section. The equatorial plane runs horizontally. The greyscale indicates the gas density (left panel), pressure (middle), and the absolute value of the velocity (right). The grid size is $200 \times 290$, adiabatic index $\gamma=1.1$, inner disk tilt $30^{\circ}$, wedge opening angle $8^{\circ}$, wind speed 40 , wind density 1 , inner sphere radius 0.05 , disk density 20 , background atmosphere density 1 .

The most extreme multipolars are formed when the inner disk is tilted between 15 and 45 degrees with respect to the equatorial plane, is thin (opening angle between 8 and 15 degrees), has a moderate density contrast (10-50 times denser than the wind), and when the fast wind has a high Mach number (20-100). "Energy-driven" shells (with $\gamma=5 / 3$ ) remain well-rounded, and never develop into the spectacular starfish-shaped nebulae that are produced when the Poisson index is close to unity. Therefore, I conclude that my mechanism will not work unless the gas is highly compressible (due to cooling), as is indeed observed, and as is required for the thermally driven disk warping. This tallies with the observers' contention (e.g. Sahai 2003) that these multipolar flows all arise during the early, dense phases of mass ejection, even before the fastest final phases of the white dwarf wind have begun.

Further exploration of parameter space showed that the outward increase of the height of the MBP-disk, which is proportional to its radius, is not ideal for the formation of multipolars. The authors argue (Maloney et al. 1996) that this unbounded increase is unrealistic anyway. I experimented with disks that have central cross sections of the form

$z=\frac{r}{a r^{2}+c} \sin (\phi+b \sqrt{r})$

(Fig. 1, right). These yielded multipolar lobes that were usually more collimated and elongated than the strict MBP cross section did (Fig. 3).

The wavy disks of the above types have equatorial cross sections that are leading spirals of the form $\phi=-b \sqrt{r}$. To complete the proof-of-principle, I have also computed the behaviour of the outflow in the equatorial plane. In that case, too, spectacular point-symmetric nebulae developed (Fig. 4).

\section{The mechanism}

Having inspected a large sample of interactions between a warped disk and a spherical wind, I can summarize the main mechanism for the formation of the multipolar lobes as follows. The central wind impinges on the inner rim of the disk. Around this, a bow shock develops, with an opening angle 


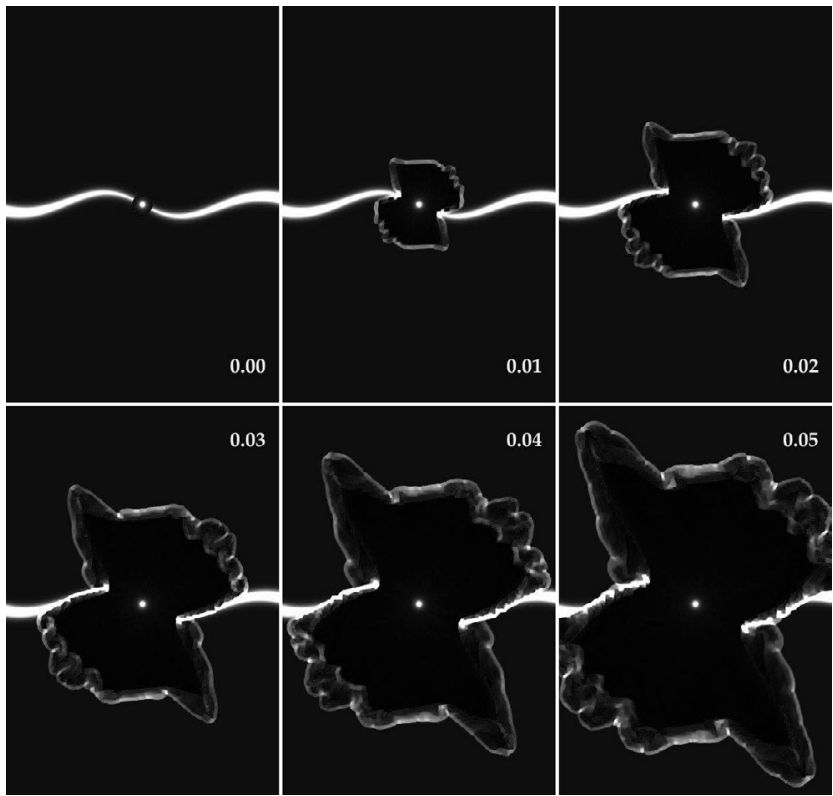

Fig. 3. Sequence of hydro frames from a simulated interaction between a fast wind and a "warped wedge" with cross section obeying Eq. (2). The equatorial plane runs horizontally, and the greyscale shows the gas density. The numbers in the boxes indicate dimensionless time. Grid size $1 \times 1.475(400 \times 590$ cells $)$, adiabatic index $\gamma=1.1$, inner disk tilt $60^{\circ}$, wedge opening angle $4^{\circ}$, wind speed 40 , wind density 1 , inner sphere radius 0.05 , disk density 20 , background atmosphere density 1 .

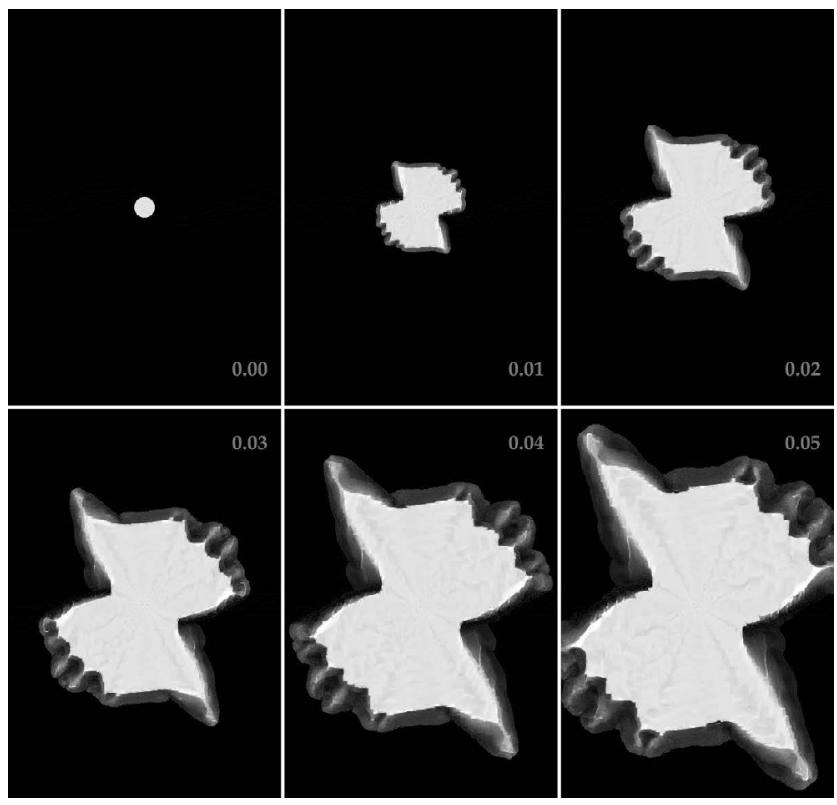

Fig. 4. As Fig. 3, but with the greyscale showing the absolute value of the velocity.

that is narrow when the central Mach number is high. One branch of this shock flies off into space, whereas the other slams into the concave side of the disk. In a strongly compressible "momentum-driven" flow, the "free" branch of the shock forms a lobe jutting out from the nebula, while the "deflected" branch scoops up disk matter and produces a set of smaller, unstable lobes. Ultimately, when the disk density is

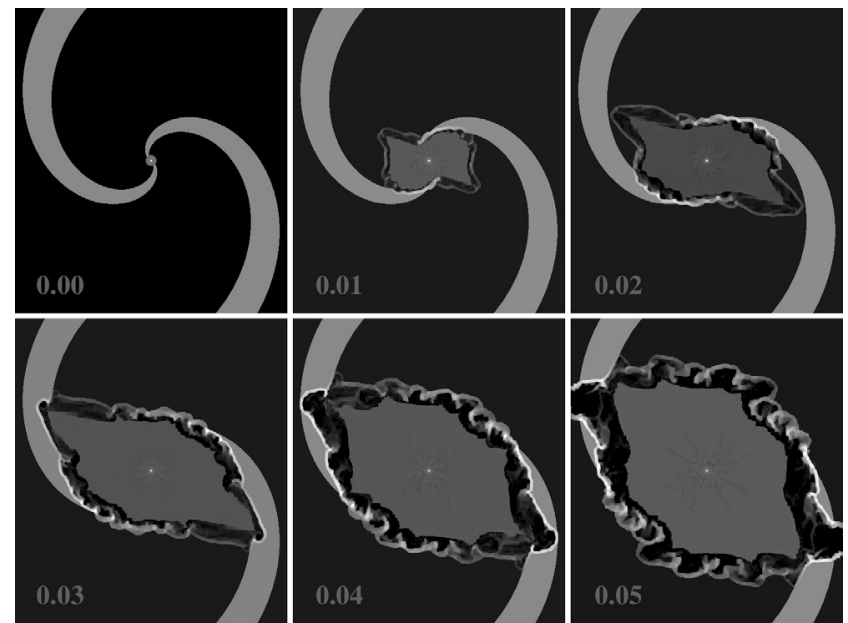

Fig. 5. Sequence of hydro frames from a simulated interaction between a fast wind and the equatorial section of the model shown in Fig. 3. The greyscale shows the gas density. The numbers in the boxes indicate dimensionless time. Grid size $1 \times 1.12(500 \times 560$ cells $)$, adiabatic index $\gamma=1.1$, wedge opening angle $25^{\circ}$, wind speed 40 , wind density 1 , inner sphere radius 0.02 , disk density 20 , background atmosphere density 1 .

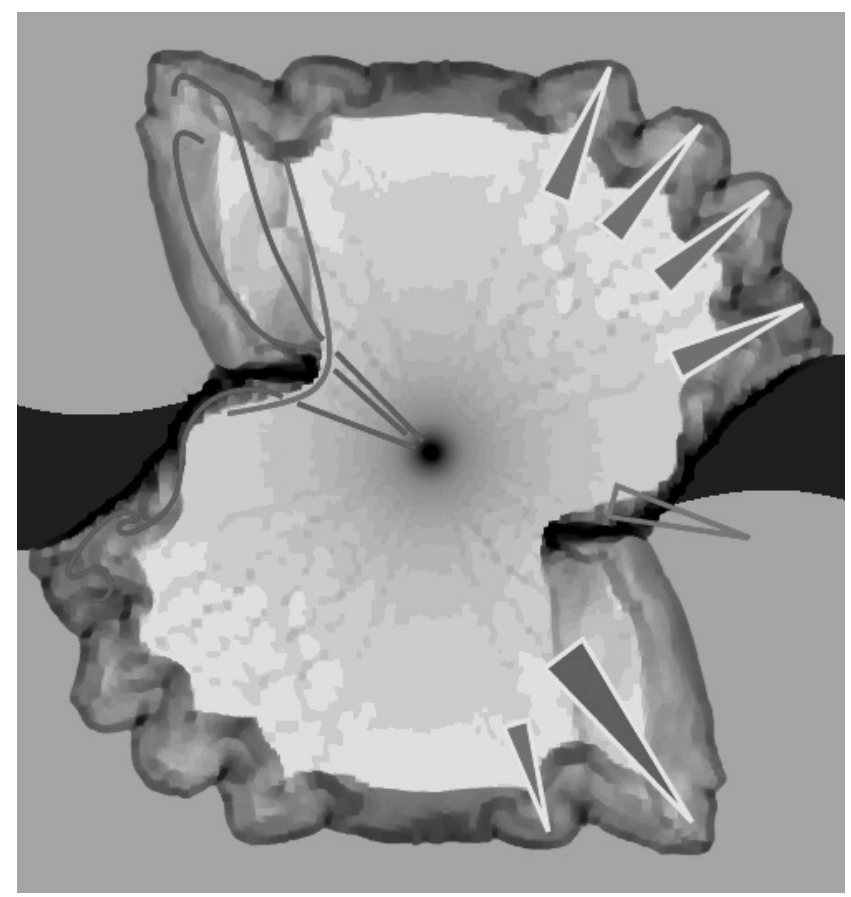

Fig. 6. Mechanism responsible for the main episode of pointsymmetric nebula formation. The background shows a greyscale map of the logarithm of the gas density in a simulation with the disk type of Eq. (2), with a rather large wedge angle $\left(25^{\circ}\right)$, producing a disk that is fairly fat at larger radii. The big wedge shows the main protrusion; smaller wedges show the secondary unstable lobes. The open wedge shows the place where the disk will be breached by the wind. The hyperbolic curve indicates the bow shock surrounding the remains of the inner rim, while the curly curves sketch the gas flow.

not too high (up to 150 times the wind density), the wind breaks through the concave part of the disk, producing yet another pair of lobes running behind the first set. However, the timing and 
appearance of this phase depend sensitively on the wedge angle and the inner tilt of the disk.

\section{Conclusions}

My computations show that the seemingly enigmatic pointsymmetric forms can be easily reproduced by a two-wind model in which the confining disk is warped. Nebulae that show "punched holes", such as the planetary nebula NGC 7027 (Cox et al. 2002), are readily accommodated in this model. As a further application, large-scale explosions in non-planar disks, such as might occur in active galaxies, are expected to show similar patterns, provided that the disk material can cool rapidly enough. It would be interesting to see if such patterns can be observed. A case in point is probably NGC 6537 (and, to a lesser extent, NGC 7026), in which the inner disk is still visible, while (in retrospect) the outer nebula shows clear pointsymmetric structures.

Acknowledgements. I thank Raghvendra Sahai for formulating the challenge that led to this paper, Garrelt Mellema and Erik-Jan Rijkhorst for their support, and Hugo Schwarz for spirited discussions.

\section{References}

Balick, B. 1987, AJ, 94, 671

Balick, B., \& Frank, A. 2002, ARA\&A, 40, 439
Boris, J. P., \& Book, D. L. 1973, J. Computat. Phys., 11, 38

Boris, J. P., \& Book, D. L. 1976, J. Computat. Phys., 20, 397

Cox, P., Huggins, P. J., Maillard, J.-P., et al. 2002, A\&A, 384, 603

García-Segura, G., Langer, N., Różyczka, M., \& Franco, J. 1999, ApJ, 517,767

Icke, V. 1988, A\&A, 202, 177

Icke, V. 1991, A\&A, 251369

Icke, V., Mellema, G., Balick, B., Eulderink, F., \& Frank, A. 1992, Nature, 355, 524

Icke, V., Preston, H. L., \& Balick, B. 1989, AJ, 97, 462

Iping, R. C., \& Petterson, J. A. 1990, A\&A, 239, 221

Livio, M., \& Pringle, J. E. 1996, ApJ, 465, L55

Livio, M., \& Pringle, J. E. 1997, ApJ, 486, 835

Maloney, P. R., Begelman, M. C., \& Pringle, J. E. 1996, ApJ, 472, 582

Mellema, G., Eulderink, F., \& Icke, V. 1991, A\&A, 252, 718

Petterson, J. A. 1977, ApJ, 214, 550

Pringle, J. E. 1996, MNRAS, 281, 357

Sahai, R. 2003, in Planetary Nebulae, ed. S. Kwok, et al., IAU Symp., 209

Sahai, R., \& Trauger, J. T. 1998, AJ, 116, 1357

Schwarz, H. E. 1993, in Mass loss on the AGB and beyond, ed. H. E. Schwarz, ESO Conf. Wrksh. Proc., 46, 223

Soker, N., \& Livio, M. 1989, ApJ, 339, 268

Soker, N., \& Livio, M. 1994, ApJ, 421, 219

Vlemmings, W. 2002, Circumstellar maser properties through VLBI observations, Ph.D. Thesis, Leiden

Vlemmings, W. H. T., Diamond, P. J., \& van Langevelde, H. J. 2002, A\&A, 394, 589 